\title{
Dynamo Mechanism of the Sun with Reference to Magnetohydrodynamic Flows
}

\author{
Swapan Kumar Ghosh \\ Department of Mathematics, Narajole Raj College, Narajole, India \\ Email: g_swapan2002@yahoo.com
}

Received 28 January 2014; revised 26 February 2014; accepted 21 March 2014

Copyright (C) 2014 by author and Scientific Research Publishing Inc.

This work is licensed under the Creative Commons Attribution International License (CC BY). http://creativecommons.org/licenses/by/4.0/

(c) (i) Open Access

\begin{abstract}
Resonance exhibits a dynamo mechanism of the Sun with an exotic behavior of a controlled thermonuclear fusion reaction in the presence of a magnetic mirror. A charged oscillator takes place of a periodic driving force so that the laser radiation is so intense with the growing of a magnetic field towards the resonant level. Following the representation of a new era of a magnetic field, it has many practical applications of magnetohydrodynamics. In response to the practical situation of interest, a mechanism has been developed with reference to a dynamo mechanism of the system.
\end{abstract}

\section{Keywords}

MHD Flow, Radio Emission, X-Emission, Laser Radiation, Displacement Current, Dynamo Mechanism, Resonance

\section{Introduction}

The configuration of the Sun is associated with thermonuclear reactor in the presence of a magnetic mirror with a decisive importance to a radiofrequency accelerator. A magnetic mirror with the Sun leads to a controlled thermonuclear fusion reaction at the resonant level and a radiofrequency accelerator with the Sun is subjected to a driving force to produce radio emission so that the emission of ultrarelativistic electron becomes gyrating in a magnetic field. This situation reveals that radio emission is a synchroton radiation. Since the Sun is exposed to a vacuum, a radio signal from the Sun is propagated into the atmosphere taking into account of X-emission. It is worth mentioning that radio emission in a vacuum communicates these radio observations to become very hottest, indeed relativistic, plasma in the universe. Plasma induced laser radiation with the Sun is subjected to a magnetic mirror in the presence of a charged oscillator with the decisive importance to a driving force. The influence of a magnetic field with the Sun communicates laser radiation at the resonant level as the magnetic field grows towards the resonant level. Ghosh [1] and Ghosh et al. [2] [3] studied the magnetohydrodynamic flow in 
a rotating environment subject to a forced oscillation in the presence of inclined magnetic field showing the influence of dynamo context of the Sun at the resonant level. Representing the influence of a magnetic field, it is stated that resonance exhibits a controlled thermonuclear fusion reaction of the Sun in the presence of a radiofrequency accelerator permeated by a magnetic mirror due to a driving force to produce laser rainbow resulting from an oscilloscope with reference to the growing of a magnetic field at the resonant level. X-ray emission is the best way of communication of laser rainbow color in such a way that the synchronization of ultraviolet radiation and $\mathrm{X}$-emission at the shorter wave length produce rainbow color in the presence of an oscilloscope and the importance of bright blue color leads to the existence of X-emission. The representation of X-ray wave in the universe deals with X-ray photon if a radiofrequency accelerator is applied to the Sun so that frictional layer of the Sun breaks down and the Sun is exposed to a vacuum. In the presence of a radiofrequency accelerator with the Sun, radio emission determines irregular fluctuation of electron in a vacuum to produce artificial rain fall. In this situation, radio emission becomes a synchronization of X-ray wave in the universe and a synchronized electromagnetic wave (X-ray photon) is liberated from the Sun.

\section{Recent Activities of the Sun}

In compliance with the study of Ghosh et al. [2] it is stated that resonance exerts its influence of dynamo mechanism of the Sun taking into account of a strong magnetic field with the Sun. The intensification of $\alpha$-effect is associated with large amplitude of the response of velocity field at resonance. Rotation by itself keeps this response at a low level, and thus acts as a constraint on the motion. The growing magnetic field at an appropriate level is to release this constraint so that the trigger large velocity fluctuation is observed. Ghosh et al. [2] studied the magnetohydrodynamic flow in a rotating environment in the presence of inclined magnetic field subject to a forced oscillation with a decisive importance to an oscillator. In the light of an angular frequency of oscillation it is rigorously stated that the resonant condition $\omega>\frac{1}{2} \cos \omega T\left(16 K^{4}-M^{4} \sin ^{4} \theta\right)^{1 / 2}$ corresponds to a forcing wave to excite the natural frequency when the phase angle $\omega T=\pi / 2$. This proves resonance when the phase angle $\omega T=\pi / 2$ so that the excitation frequency becomes relevant to $\omega>0$. In this context, a magnetic mirror with the Sun will generate an excitation frequency to produce laser radiation due to a driving force in the presence of a charged oscillator. This situation reveals that laser radiation is so intense at the transition when the phase angle $\omega T=\pi / 2$ at resonance and the reflection occurs in the region as the magnetic field increases abruptly in strength. It is strictly speaking that a magnetic mirror with the Sun is the representation of an inclined magnetic field to produce dynamo mechanism of the Sun at resonant level. The excitation frequency $\omega>0$ due to a driving force permeated by a radiofrequency accelerator leads to a turbulent dynamo mechanism of the Sun in the presence of a magnetic mirror. The stability of a magnetohydrodynamic system of the Sun is subjected to the simultaneous action of Coriolis and Lorentz force whereas the effect of rotation $\left(K^{2}\right)$ and magnetic force $\left(M^{2}\right)$ are separately stabilizing, the two effects can work against each other in such a way that the flow is stable under the action of rotation alone and the flow becomes unstable when the magnetic field is present. It is worth mentioning that the resonant condition $\omega>\frac{1}{2} \cos \omega T\left(16 K^{4}-M^{4} \sin ^{4} \theta\right)^{1 / 2}$ leads to a turbulent dynamo mechanism of the Sun where $K^{2}$ to the rotation parameter which is the reciprocal of Ekman number, $M^{2}$ is the Hartmann number (magnetic force) and $\theta$ is the angle of inclination of a magnetic field with the positive direction of the axis of rotation. In the presence of a charged oscillator with the Sun, the turbulent dynamo mechanism corresponds to an oscillatory character of the Sun at the resonant level. The trigger large velocity fluctuation communicates with the growing of a magnetic field at the resonant level to exhibit a turbulent dynamo context of the sun if the Coriolis force and magnetic force are comparable in magnitude. Representing the charged particle in the presence of a radiofrequency accelerator with the Sun, the formation of electron cloud particle exerts its influence of a controlled thermonuclear fusion reaction of the Sun permeated by a magnetic mirror. This situation reveals that radio emission determines the artificial rain fall so that irregular fluctuation of hot election is liberated from the Sun due to the presence of a strong magnetic field. It is important to note that the turbulent dynamo mechanism of the Sun leads to a stability criteria with the growing of a magnetic field to release the constraint at the resonant level so that resonance exhibits a symmetric chao with reference to a dynamo context of the universe.

In relevance to the physical situation of interest, Ghosh et al. [3] have extended the problem on unsteady 
magnetohydrodynamic free and forced convection flow in the presence of inclined magnetic field subject to forced oscillation. A charged oscillator takes place of a driving force in the presence of a magnetic mirror to exhibit resonance under the condition $\omega>\frac{1}{2} \cos \omega T\left(16 K^{4}-M^{4} \sin ^{4} \theta\right)^{1 / 2}$. The convective part of the surface of the Sun in the presence of a magnetic mirror deals with intense laser radiation with reference to a charged oscillator so that the magnetic field line changes its direction abruptly from the central region. This situation reveals that the growth of a magnetic field toward the resonant level exceeds the critical value taking into account of a differential rotation of the Sun which becomes a remarkable evidence of a cyclonic turbulence. Cyclonic turbulence generally happens in the case of an increase in differential rotation of the Sun when a forced oscillation is taken into account. Since the magnetic field lines change their direction abruptly from the central region of the convective part of the Sun, the differential rotation of the Sun increases with the growing of a magnetic field towards the resonant level which is the most powerful evidence of solar storm. This indicates that the charged particle is propagated from the Sun to form a solar storm with reference to an abrupt increase in magnetic field so that irregular fluctuations of hot electrons emitted irregularly with an abrupt change of magnetic field lines from the central region. Owing to convective part of the surface of the Sun under the influence of a magnetic mirror, a charged oscillator takes place of an external periodic driving force in such a way that a laser pulse radiation is emitted from the Sun which lies in its behavior of a photon under resonant condition to a dynamo context of the Sun as the magnetic field determines resonance fluorescence when the phase angle $\omega T=\pi / 2$ with regard to the excitation frequency $\omega>0$. This proves that a synchronized photon light (Laser pulse radiation) is emitted from the Sun.

In relevance to the study of Ghosh [1] and Ghosh et al. [2] [3] the physical existence of these problems can be established with regard to $\nabla . J \neq 0$ where $J$ is the current density vector. All these studies lead to a displacement current effect due to an external periodic driving force subject to a forced oscillation in the presence of an inclined magnetic field with regard to $\nabla . J \neq 0$. When a displacement current is taken into account, it is possible to consider even those situations which involve non-steady currents i.e. situations where $\nabla . J \neq 0$. Ac circuits furnish a special case of time varying electromagnetic phenomenon, where all the variations are sinusoidal in character. The action of such circuits with capacitors as circuit elements can be understood on the basis of the displacement current. The usage of the term displacement current is considered by some authors as unfortunate and misleading. Clearly, one should not have visions of something being displaced from one point to another that gives rise to displacement current. Indeed, the difference between a steady current flow and an unsteady current flow is that $\nabla . J$ is zero for the former while it is non-zero for the latter.

\section{Practical Field of Interest}

With a view to analyze the practical importance of all these studies may have a significant feature of dynamo mechanism of the system to deal with resonance radiation. This implicates the situation of a rotating environment by employing a periodic driving force under the influence of a charged oscillator in the presence of a magnetic mirror to exhibit intense laser radiation which has many practical applications of heavy solid metal melting process as well as fighter plane crash. The mechanism of such applications can be developed in such a way that an AC circuit furnish with a coil surrounding the drum with reference to a capacitor showing the time varying electromagnetic phenomenon is subjected to a electrical relay analogy where all the variation are sinusoidal in character in the presence of a charged oscillator. Following a device can be introduced in terms of a magnetic mirror so that the dynamo mechanism of a magnetic field line leads to resonance in the presence of a periodic driving force. In such a situation, the angular frequency and phase angle can be related to each other so that the system rotates with an angular frequency $\omega$ and maximum reflection occurs when the phase angle $\omega T=\pi / 2$ at resonance. The growing of a magnetic field towards the resonant level is associated with maximum reflection to hit the target of a fighter plane as the magnetic field increases abruptly in strength. Representing the new era of a magnetic field it is a decisive importance to many areas of magnetohydrodynamics to deal with different flow situations.

\section{Conclusion}

Turbulent dynamo mechanism of the Sun at the resonant level emerges from the back bone of a strong magnetic field in the presence of a magnetic mirror with a decisive importance to a pulse oscillator. A charged oscillator 
takes place of a driving force to produce laser pulse radiation (photon light) in the presence of a magnetic mirror. Representing the turbulent dynamo mechanism of the Sun at the resonant level it is subjected to say that a kite is a dynamo in relation with a new era of magnetic field if the magnetic force and Coriolis force are comparable of magnitude. In relevance to the practical situation of interest, a mechanism has been developed with reference to intense laser radiation at resonance.

\section{References}

[1] Ghosh, S.K. (2001) A Note on Unsteady Hydromagnetic Flow in a Rotating Channel Permeated by an Inclined Magnetic Field in the Presence of an Oscillator. Czechoslovak Journal of Physics, 51, 799-804. http://dx.doi.org/10.1023/A:1011622416362

[2] Ghosh, S.K., Beg, O.A., Zueco, J. and Prasad, V.R. (2010) Transient Hydromagnetic Flow in a Rotating Channel Permeated by an Inclined Magnetic Field with Magnetic Induction and Maxwell Displacement Current Effects. Zeitschrift fur Angewandte Mathematik und Physik ( ZAMP), 61, 147-169. http://dx.doi.org/10.1007/s00033-009-0006-2

[3] Ghosh, S.K., Anwar Beg, O. and Narahari, M. (2013). A Study of Unsteady Rotating Hydromagnetic Free and Forced Convection in a Channel Subject to Forced Oscillation under an Oblique Magnetic Field. Journal of Applied Fluid Mechanics, 6, 213-227. 